\title{
Statyba
}

\section{DUAL RELATIONS OF LINEAR THIN GENERAL SHELL THEORY}

\section{R. Karkauskas}

To cite this article: R. Karkauskas (1997) DUAL RELATIONS OF LINEAR THIN GENERAL

SHELL THEORY, Statyba, 3:9, 66-73, DOI: 10.1080/13921525.1997.10531673

To link to this article: https://doi.org/10.1080/13921525.1997.10531673

曲 Published online: 26 Jul 2012.

Submit your article to this journal $2 \pi$

山 Article views: 44 


\section{DUAL RELATIONS OF LINEAR THIN GENERAL SHELL THEORY}

\section{R. Karkauskas}

\section{Some fundamentals}

We use the "classical" shell theory based upon the Love and Kirchhoff approximate assumption, that straight lines normal to the middle surface before deformation remain straight, normal to the middle surface and unchanged in length after deformation.

The errors due to this approximation are negligible for "thin shells" whose wall thickness is small compared to the "radius of curvature" $R_{\min }$ $\left(h / R_{\min } \leq 1 / 20\right)$. On the other hand, we know, that for thin shells the normal stress $\sigma_{z}$ is small compared to other normal stresses. Thus the shell wall is in a condition of plane stress.

As coordinate lines we will use the "lines of curvature" of a continuous curved surface such as the undisplaced middle surface of the shell wall, together with normals to this surface. These lines of curvature are defined as lines along which the twist is zero. It is shown in the theory of continuous surfaces that there are always at least two such systems of lines, and that these systems are orthogonal to each other, that is tangents to two such lines at the point where they intersect will be at right angles to each other. Fig. 1 shows a point $o$ with the orthogonal lines of curvature labelled $\alpha_{1}$ and $\alpha_{2}$, passing through $o$. We take the coordinates of point $o$ as $\alpha_{1}, \alpha_{2}$ and of the points, $a, b$, adjacent to $o$ in the directions of increase of $\alpha_{1}, \alpha_{2}$, as $\alpha_{1}+d \alpha_{1}, \alpha_{2}$ and $\alpha_{1}, \alpha_{2}+d \alpha_{2}$ respectively, as indicated (Fig. 1). We introduce variable scale factors $A_{1}, A_{2}$ defined so that $A_{1} d \alpha_{1}$ and $A_{2} d \alpha_{2}$ are the distances measured along the curves between $o$ and $a$ and between $O$ and $b$; we assume $A_{1}, A_{2}$ to be continuous functions of $\alpha_{1}, \alpha_{2}$. These functions are called "coefficients of the first quadratic form" of continuous surfaces. Let the equations of middle surface be completely described by the expressions:

$$
x=x\left(\alpha_{1}, \alpha_{2}\right), \quad y=y\left(\alpha_{1}, \alpha_{2}\right), \quad z=z\left(\alpha_{1}, \alpha_{2}\right) .
$$

Thus for defining $A_{1}, A_{2}$ we have:

$$
\begin{aligned}
& A_{1}=\sqrt{\left(\alpha x / \partial \alpha_{1}\right)^{2}+\left(\partial / \partial \alpha_{1}\right)^{2}+\left(\partial z / \partial \alpha_{1}\right)^{2}}, \\
& A_{2}=\sqrt{\left(\alpha / \partial \alpha_{2}\right)^{2}+\left(\partial / \partial \alpha_{2}\right)^{2}+\left(\alpha z / \partial \alpha_{2}\right)^{2}} .
\end{aligned}
$$

We next erect rectangular coordinate axes $x, y, z$ with origin at $o$, taking the $x$ and $y$ axes tangent respectively to the orthogonal $\alpha_{1}, \alpha_{2}$ lines at $o$, as shown in Fig. 1. They are fixed axes, because the $\alpha_{1}$, $\alpha_{2}$ lines are fixed in the undisplaced middle surface.

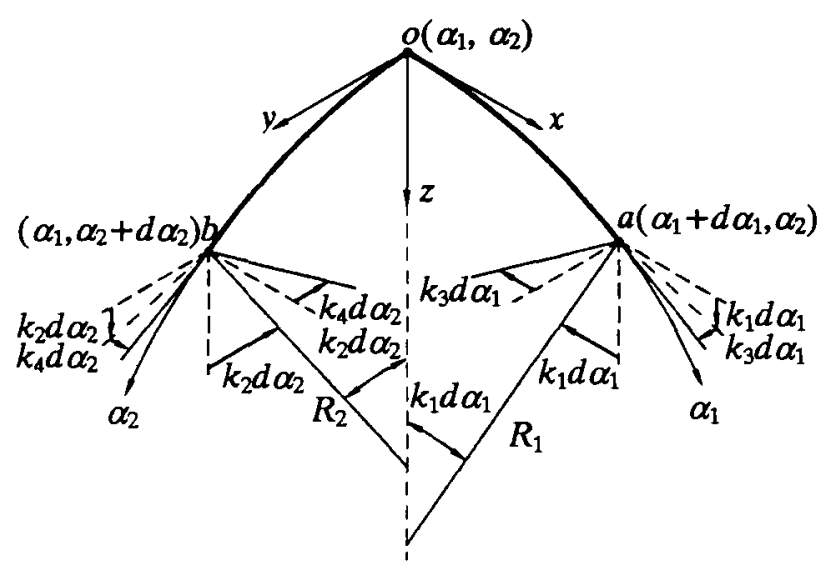

Fig. 1. Original positions

Similar triads of axes are erected at points $a$ and $b$; they are indicated in Fig. 1 by the full straight lines through $a$ and $b$. Due to the curvature of the surface and of the $\alpha_{1}, \alpha_{2}$ coordinate lines in the surface these triads will, in general, be rotated relative to the $x y z$ directions, that is relative to the dotted lines shown in the figure.

These angles of rotation about the $x y z$ directions are shown in Fig. 1. We take the rotations of the triad at $a$ about the $x y z$ directions to be $0, k_{1} d \alpha_{1}, k_{3} d \alpha_{1}$ respectively, as shown. Similarly, the triad at $b$ is 


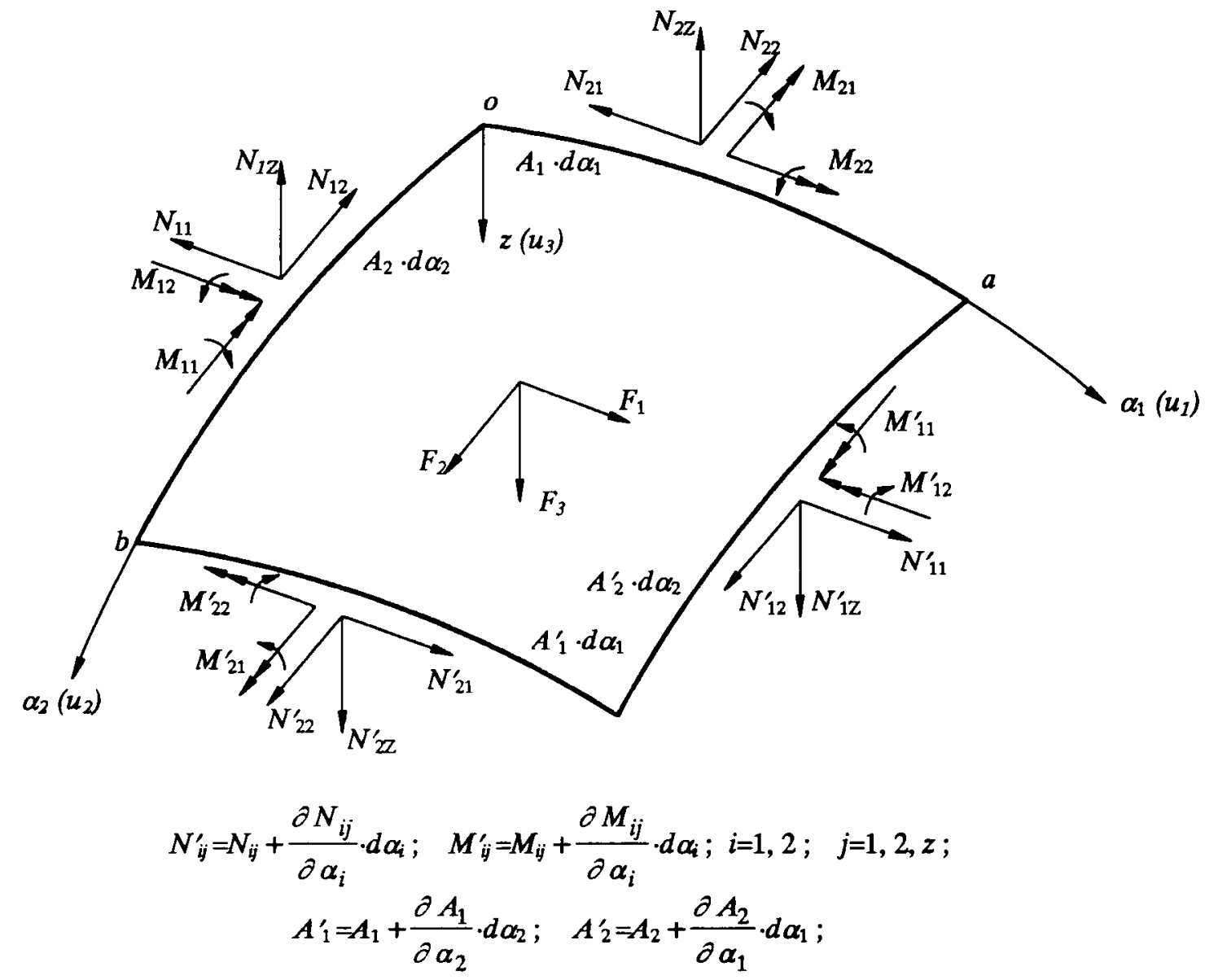

Fig. 2. Total forces and total moments on element sides

rotated through the angles $k_{2} d \alpha_{2}, 0, k_{4} d \alpha_{2}$ about the $x y z$ directions, as shown. $k_{1}, k_{2}, k_{3}$ and $k_{4}$ are assumed to be continuous functions of $\alpha_{1}, \alpha_{2}$ having the physical meanings of a curvature of the surface in the $\alpha_{1}, \alpha_{2}$ directions and of the $\alpha_{1}, \alpha_{2}$ lines in the surface. Altogether it can be shown ([1]) that:

$$
\begin{array}{ll}
k_{1}=A_{1} / R_{1}, & k_{2}=A_{2} / R_{2}, \\
k_{3}=-\frac{1}{A_{2}} \frac{\partial A_{1}}{\partial \alpha_{2}}, & k_{4}=-\frac{1}{A_{1}} \frac{\partial A_{2}}{\partial \alpha_{1}} .
\end{array}
$$

These relations will be found useful.

\section{General shell equilibrium equations}

The equilibrium equations are found by considering the equilibrium of an infinitesimal element of a continuous curved surface, of the dimensions $A_{1} d \alpha_{1}$ and $A_{2} d \alpha_{2}$, as illustrated in Fig. 2. For simplicity, we show only the undisplaced middle surface of the shell, with all the forces acting on the element and the moments about the middle surface.
We define the N's and M's shown as forces and moments per unit length of section at the middle surface. The forces and moments are designated by two subscripts, the first of them indicates the direction of the normal to the side on which the stresses act, and the second the direction of the axis.

The components of the external distributed load which act on the surface are shown in Fig. 2; $F_{f}\left(\alpha_{1}, \alpha_{2}\right)$, $j=1,2,3$ is a distributed force per unit area acting in the positive direction of the axis. To the approximation which we obtain by using the Love - Kirchhoff assumption, we can not distinguish between such a force acting on the upper or lower surface.

The information given in Figs. 1 and 2 can now be used to write down the six equations of equilibrium. We need equations of equilibrium of forces in the $\alpha_{1}, \alpha_{2}, z$ directions $\sum F_{\alpha_{1}}, \sum F_{\alpha_{2}}$, $\sum F_{z}=0$ and of moments of the forces about axes in the $\alpha_{1}, \alpha_{2}, z$ directions, say, through the centre of the element, $\sum M_{\alpha_{1}}, \sum M_{\alpha_{2}}, \sum M_{z}=0$. 
From the above discussion, it is evident that total forces equilibrium in the $\alpha_{1}$ directions requires that:

$$
\begin{aligned}
& -N_{11} A_{2} d \alpha_{2}+N_{11}^{\prime} A_{2}^{\prime} d \alpha_{2}-N_{21} A_{1} d \alpha_{1}+N_{21}^{\prime} A_{1}^{\prime} d \alpha_{1} \\
& +N_{22}^{\prime} A_{1}^{\prime} d \alpha_{1} k_{4} d \alpha_{2}-N_{12}^{\prime} A_{2}^{\prime} d \alpha_{2} k_{3} d \alpha_{1} \\
& \quad-N_{12}^{\prime} A_{2}^{\prime} d \alpha_{2} k_{1} d \alpha_{1}-F_{1} A_{1} d \alpha_{1} A_{2} d \alpha_{2}=0 .
\end{aligned}
$$

Using information given in Fig. 2 and Eqs. (2) and dividing by the factor $d \alpha_{1} d \alpha_{2}$ (terms containing more increments of course being ignored as small quantities of higher order), the first equation is presented:

$$
\begin{aligned}
\frac{\partial\left(A_{2} N_{11}\right)}{\partial \alpha_{1}}+\frac{\partial\left(A_{1} N_{21}\right)}{\partial \alpha_{2}}+k_{4} A_{1} N_{22}-k_{3} A_{2} N_{12} & \\
& -k_{1} A_{2} N_{1 z}+F_{1} A_{1} A_{2}=0 .
\end{aligned}
$$

The second equation is the same as this with subscripts 1 and 2,3 and 4 interchanged:

$$
\begin{aligned}
\frac{\partial\left(A_{1} N_{22}\right)}{\partial a_{2}}+\frac{\partial\left(A_{2} N_{12}\right)}{\partial \alpha_{1}}+k_{3} A_{2} N_{11}-k_{4} A_{1} N_{21} \\
-k_{2} A_{1} N_{2 z}+A_{2} A_{1} F_{2}=0 .
\end{aligned}
$$

The third equilibrium equation (the most important of them all, since it represents equilibrium of forces tending to deform the shell in its weakest direction, the direction of the small thickness) for the $z$ direction is:

$-N_{1 z} A_{2} d \alpha_{2}+N_{1 z}^{\prime} A_{2}^{\prime} d \alpha_{2}-N_{2 z} A_{1} d \alpha_{1}+N_{2 z}^{\prime} A_{1}^{\prime} d \alpha_{1}$

$+N_{11}^{\prime} A_{2}^{\prime} d \alpha_{2} k_{1} d \alpha_{1}+N_{22}^{\prime} A_{1}^{\prime} d \alpha_{1} k_{2} d \alpha_{2}$

$$
+F_{3} A_{1} d \alpha_{1} A_{2} d \alpha_{2}=0
$$

Cancelling $d \alpha_{1} d \alpha_{2}$ and using information given in Fig. 2, these can be written in the form:

$$
\begin{aligned}
k_{1} A_{2} N_{11}+k_{2} A_{1} N_{22}+\frac{\partial\left(A_{2} N_{1 z}\right)}{\partial \alpha_{1}}+\frac{\partial\left(A_{1} N_{2 z}\right)}{\partial \alpha_{2}} & \\
& +A_{1} A_{2} F_{3}=0 .
\end{aligned}
$$

The fourth equation of equilibrium, stating that the moments of forces about the $\alpha_{2}$ direction up to zero, becomes:

$$
\begin{aligned}
\frac{\partial\left(A_{2} M_{11}\right)}{\partial \alpha_{1}}+\frac{\partial\left(A_{1} M_{21}\right)}{\partial \alpha_{2}}+k_{4} A_{1} M_{22}- & k_{3} A_{2} M_{12} \\
& -A_{1} A_{2} N_{1 z}=0 .
\end{aligned}
$$

$$
\begin{aligned}
& \frac{\partial\left(A_{1} M_{22}\right)}{\partial \alpha_{2}}+\frac{\partial\left(A_{2} M_{12}\right)}{\partial \alpha_{1}}+k_{3} A_{2} M_{11}-k_{4} A_{1} M_{21} \\
&-A_{2} A_{1} N_{2 z}=0 .
\end{aligned}
$$

The last equation of equilibrium, of moments about the $z$ axis, becomes:

$$
\frac{k_{1}}{A_{1}} M_{12}-\frac{k_{2}}{A_{2}} M_{21}+N_{21}-N_{12}=0 .
$$

This equation is an identity. It will be seen that as we have the integral expressions of the initial forces:

$$
\begin{aligned}
& \frac{k_{1}}{A_{1}} \int_{-0.5 h}^{0.5 h} \sigma_{12}\left(1-\frac{k_{2}}{A_{2}} z\right) z d z-\frac{k_{2}}{A_{2}} \int_{-0.5 h}^{0.5 h} \sigma_{21}\left(1-\frac{k_{1}}{A_{1}} z\right) z d z \\
& +\int_{-0.5 h}^{0.5 h} \sigma_{21}\left(1-\frac{k_{1}}{A_{1}} z\right) d z-\int_{-0.5 h}^{0.5 h} \sigma_{12}\left(1-\frac{k_{2}}{A_{2}} z\right) d z \\
& =\int_{-0.5 h}^{0.5 h}\left(\sigma_{21}-\sigma_{12}\right)\left(1-\frac{k_{1}}{A_{1}} z\right)\left(1-\frac{k_{2}}{A_{2}} z\right) d z=0
\end{aligned}
$$

because $\sigma_{21}=\sigma_{12}$.

We can now solve for $N_{1 z}$ and $N_{2 z}$ from the fourth and fifth equations, which represent equilibrium of moments about the $\alpha_{2}$ and $\alpha_{1}$ directions. With these modifications and replacing $k_{1}$, $k_{2}, k_{3}, k_{4}$ by Eqs.(2), the general shell equilibrium equations become:

$$
\begin{array}{r}
-\frac{\partial\left(A_{2} N_{11}\right)}{\partial \alpha_{1}}+\frac{\partial A_{2}}{\partial \alpha_{1}} N_{22}-\frac{\partial A_{1}}{\partial \alpha_{2}} N_{12}-\frac{\partial\left(A_{1} N_{21}\right)}{\partial \alpha_{2}} \\
+\frac{1}{R_{1}} \frac{\partial\left(A_{2} M_{11}\right)}{\partial \alpha_{1}}-\frac{1}{R_{1}} \frac{\partial A_{2}}{\partial \alpha_{1}} M_{22}+\frac{1}{R_{1}} \frac{\partial A_{1}}{\partial \alpha_{2}} M_{12} \\
+\frac{1}{R_{1}} \frac{\partial\left(A_{1} M_{21}\right)}{\partial \alpha_{2}}=A_{1} A_{2} F_{1}
\end{array}
$$

$$
\begin{array}{r}
-\frac{\partial\left(A_{1} N_{22}\right)}{\partial \alpha_{2}}+\frac{\partial A_{1}}{\partial \alpha_{2}} N_{11}-\frac{\partial A_{2}}{\partial \alpha_{1}} N_{21}-\frac{\partial\left(A_{2} N_{12}\right)}{\partial \alpha_{1}} \\
+\frac{1}{R_{2}} \frac{\partial\left(A_{1} M_{22}\right)}{\partial \alpha_{2}}-\frac{1}{R_{2}} \frac{\partial A_{1}}{\partial \alpha_{2}} M_{11}+\frac{1}{R_{2}} \frac{\partial A_{2}}{\partial \alpha_{1}} M_{21} \\
+\frac{1}{R_{2}} \frac{\partial\left(A_{2} M_{12}\right)}{\partial \alpha_{1}}=A_{1} A_{2} F_{2}
\end{array}
$$

The fifth equation is the same as this with subscripts 1 and 2, 3 and 4 interchanged: 
Table 1. Differential operator of equilibrium equations

\begin{tabular}{|c|c|c|c|c|c|}
\hline$-\frac{\partial\left(A_{2} \cdot \ldots\right)}{\partial \alpha_{1}}$ & $\frac{\partial A_{2}}{\partial \alpha_{1}} \cdot \ldots$ & $\begin{array}{l}-\frac{\partial A_{1}}{\partial \alpha_{2}} \ldots \\
-\frac{\partial\left(A_{1} \ldots\right)}{\partial \alpha_{2}}\end{array}$ & $\frac{1}{R_{1}} \frac{\partial\left(A_{2} \cdot \ldots\right)}{\partial \alpha_{1}}$ & $-\frac{1}{R_{1}} \frac{\partial A_{2}}{\partial \alpha_{1}} \ldots$ & $\begin{array}{c}\frac{1}{R_{1}} \frac{\partial A_{1}}{\partial \alpha_{2}} \ldots \\
+\frac{1}{R_{1}} \frac{\partial\left(A_{1} \cdots\right)}{\partial \alpha_{2}}\end{array}$ \\
\hline$\frac{\partial A_{1}}{\partial \alpha_{2}}$ & $-\frac{\partial\left(A_{1} \ldots\right)}{\partial \alpha_{2}}$ & $\begin{array}{l}-\frac{\partial A_{2}}{\partial \alpha_{1}} \ldots \\
-\frac{\partial\left(A_{2} \ldots\right)}{\partial \alpha_{1}}\end{array}$ & $-\frac{1}{R_{2}} \frac{\partial A_{1}}{\partial \alpha_{2}}$ & $\frac{1}{R_{2}} \frac{\partial\left(A_{1} \cdot \ldots\right)}{\partial a_{2}}$ & $\begin{array}{c}\frac{1}{R_{2}} \frac{\partial A_{2}}{\partial \alpha_{1}} \cdot \ldots \\
+\frac{1}{R_{2}} \frac{\partial\left(A_{2} \cdot \ldots\right)}{\partial \alpha_{1}}\end{array}$ \\
\hline$-\frac{A_{1} A_{2}}{R_{1}}$. & $-\frac{A_{1} A_{2}}{R_{2}}$ & 0 & $\begin{array}{l}-\frac{\partial}{\partial \alpha_{1}}\left(\frac{1}{A_{1}} \frac{\partial\left(A_{2} \cdot \ldots\right)}{\partial \alpha_{1}}\right) \\
+\frac{\partial}{\partial \alpha_{2}}\left(\frac{1}{A_{2}} \frac{\partial A_{1}}{\partial \alpha_{2}} \ldots\right)\end{array}$ & $\begin{array}{l}-\frac{\partial}{\partial \alpha_{2}}\left(\frac{1}{A_{2}} \frac{\partial\left(A_{1} \ldots\right)}{\partial \alpha_{2}}\right) \\
+\frac{\partial}{\partial \alpha_{1}}\left(\frac{1}{A_{1}} \frac{\partial A_{1}}{\partial \alpha_{1}} \cdot \ldots\right)\end{array}$ & $\begin{array}{l}-\frac{\partial}{\partial \alpha_{1}}\left(\frac{1}{A_{1}} \frac{\partial\left(A_{1} \cdot \ldots\right)}{\partial \alpha_{2}}\right) \\
-\frac{\partial}{\partial \alpha_{2}}\left(\frac{1}{A_{2}} \frac{\partial\left(A_{2} \cdot \ldots\right)}{\partial \alpha_{1}}\right) \\
-\frac{\partial}{\partial \alpha_{1}}\left(\frac{1}{A_{1}} \frac{\partial A_{1}}{\partial \alpha_{2}} \cdot \ldots\right) \\
-\frac{\partial}{\partial \alpha_{2}}\left(\frac{1}{A_{2}} \frac{\partial A_{2}}{\partial \alpha_{1}} \cdot \ldots\right)\end{array}$ \\
\hline
\end{tabular}

$$
\begin{gathered}
-\frac{A_{1} A_{2}}{R_{1}} N_{11}-\frac{A_{2} A_{1}}{R_{2}} N_{22}-\frac{\partial}{\partial \alpha_{1}}\left(\frac{1}{A_{1}} \frac{\partial\left(A_{2} M_{11}\right)}{\partial \alpha_{1}}\right) \\
-\frac{\partial}{\partial \alpha_{1}}\left(\frac{1}{A_{1}} \frac{\partial\left(A_{1} M_{21}\right)}{\partial \alpha_{2}}\right)+\frac{\partial}{\partial \alpha_{1}}\left(\frac{1}{A_{1}} \frac{\partial A_{2}}{\partial \alpha_{1}} M_{22}\right) \\
-\frac{\partial}{\partial \alpha_{1}}\left(\frac{1}{A_{1}} \frac{\partial A_{1}}{\partial \alpha_{2}} M_{12}\right)-\frac{\partial}{\partial \alpha_{2}}\left(\frac{1}{A_{2}} \frac{\partial\left(A_{1} M_{22}\right)}{\partial \alpha_{2}}\right) \\
-\frac{\partial}{\partial \alpha_{2}}\left(\frac{1}{A_{2}} \frac{\partial\left(A_{2} M_{12}\right)}{\partial \alpha_{1}}\right)+\frac{\partial}{\partial \alpha_{2}}\left(\frac{1}{A_{2}} \frac{\partial A_{1}}{\partial \alpha_{2}} M_{11}\right) \\
-\frac{\partial}{\partial \alpha_{2}}\left(\frac{1}{A_{2}} \frac{\partial A_{2}}{\partial \alpha_{1}} M_{21}\right)=A_{1} A_{2} F_{3} .
\end{gathered}
$$

The differences between $N_{12}$ and $N_{21}$ or between $M_{12}$ and $M_{21}$ are minor quantities for thin shells and can be ignored for most purposes. In this case we would have $N_{12}=N_{21}$ and $M_{12}=M_{21}$. Then the strained state of the shell are defined by the six-dimensional vector-function of forces

$$
\mathbf{S} \equiv\left(N_{11}, N_{22}, N_{12}=N_{21}, M_{11}, M_{22}, M_{12}=M_{21}\right)^{\mathrm{T}} \text {. }
$$

These forces are of course in general functions of both $\alpha_{1}$ and $\alpha_{2}$.

The load is characterized by the threedimensional vector-function of distributed load

$$
\mathbf{F} \equiv\left(F_{1}, F_{2}, F_{3}\right)^{\mathrm{T}} \text {. }
$$

If the vectors $\mathbf{S}$ and $\mathbf{F}$ are chosen as indicated above, then the Eqs. (3) of a general shell have the form

$$
[\mathrm{A}] \mathbf{S}=\mathbf{F} .
$$

where $[\mathrm{A}]$ is the differential operator of the equilibrium equations. It is shown in Table 1.

\section{General shell geometric equations}

The geometric equations, which define the connection between displacements and deformations, can be obtained purely formally since the operators of the equilibrium equations and kinematic compatibility are adjoints. Thus we have

$$
[A]^{\mathrm{T}} \mathbf{u}=\mathbf{q} \text {. }
$$


Table 2. Differential operator of geometric equations

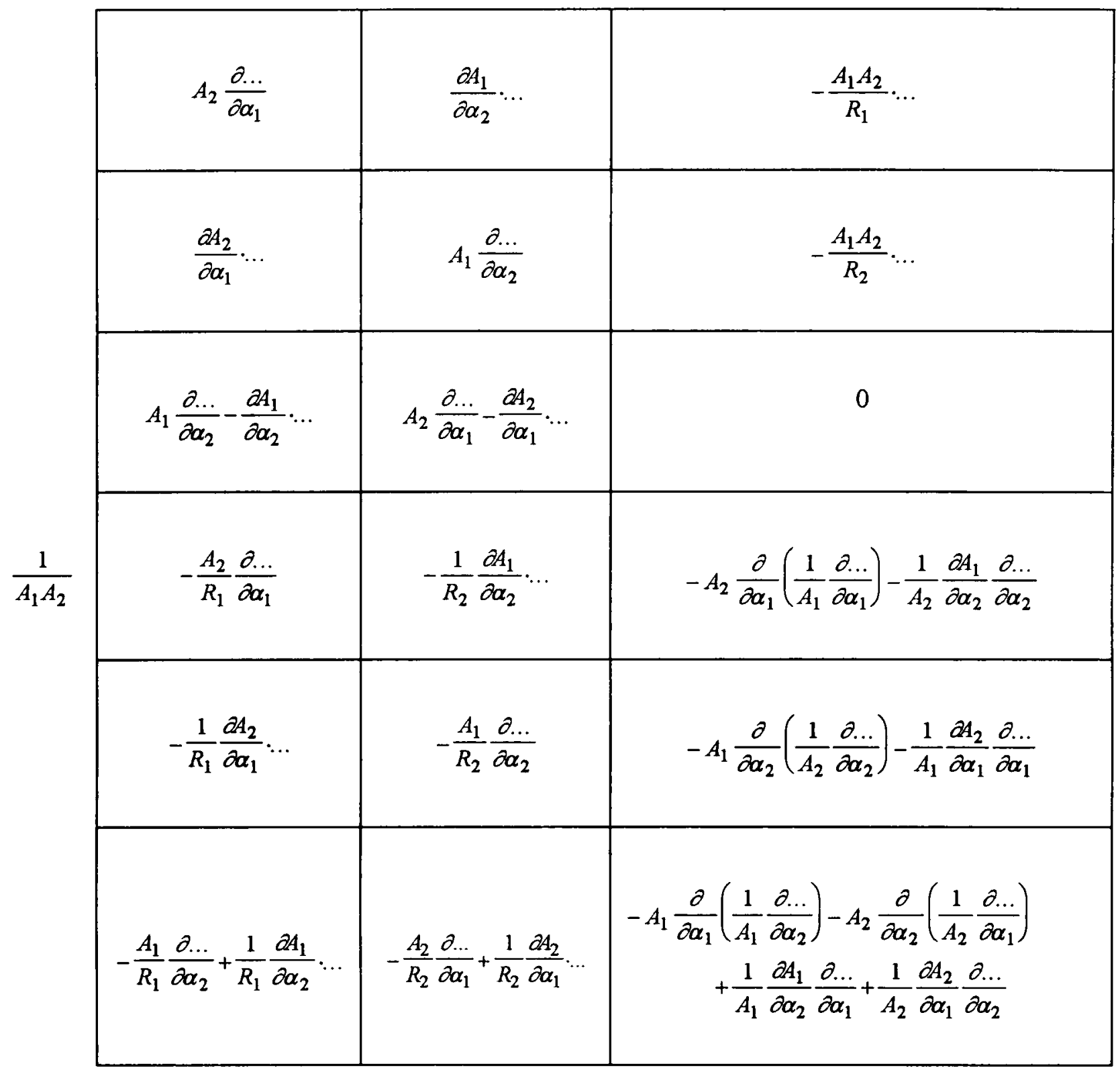

Here the vector-function $\mathbf{u}$ is the dual variable for the load $F$, and denotes the displacements. We designate the components in the $\alpha_{1}, \alpha_{2}, z$ directions of the displacements of points in the middle surface of the shell by $u_{v} u_{2}, u_{3}$, as indicated in the parentheses after the axis designations in Fig. 2. These displacements $u_{1}, u_{2}, u_{3}$ are of course in general functions of both $\alpha_{1}$ and $\alpha_{2}$. Then the vector

$$
\mathbf{u} \equiv\left(u_{1}, u_{2}, u_{3}\right)^{\mathrm{T}} \text {. }
$$

The vector-function $q$ is the dual of the vector of forces $\mathbf{S}$, and denotes the strains. We designate the components of the strains by $\Delta_{11}, \Delta_{22}, \Delta_{12}, \chi_{11}, \chi_{22}, \chi_{12}$.
The first three terms are the "membrane" strains; $\Delta_{11}, \Delta_{22}$ are the changes in length of the middle surface of an infinitesimal element in the $\alpha_{1}, \alpha_{2}$ directions, $\Delta_{12}$ is the change in the angle between the sides of element, whose normals were originally in the $\alpha_{1}$ and $\alpha_{2}$ directions. The last three terms are the "flexural" strains; $\chi_{11}, \chi_{2}$ are the curvatures of the middle surface in the $\alpha_{1} \alpha_{2}$ directions, $\chi_{12}$ is the twist of the surface.

The operator $[\mathrm{A}]^{\mathrm{T}}$ is the transpose of the operator of the equation of equilibrium. Altogether it can be shown ([2]) that: 
if $\quad a_{i j}=f\left(\alpha_{1}, \alpha_{2}\right) \frac{\partial^{m+n}\left(f\left(\alpha_{1}, \alpha_{2}\right) u_{i}\right)}{\partial \alpha_{1}^{m} \partial \alpha_{2}^{n}}$

then $a_{j i}^{\mathrm{T}}=(-1)^{m+n} \varphi\left(\alpha_{1}, \alpha_{2}\right) \frac{\partial^{m+n}\left(f\left(\alpha_{1}, \alpha_{2}\right) u_{i}\right)}{\partial \alpha_{1}^{m} \partial \alpha_{2}^{n}}$.

Using Eqs. ( 6 ), we find the operator $[\mathrm{A}]^{\mathrm{T}}$, given in Table 2. This information can now be used to write down the six equations of kinematic compatibility:

$$
\begin{aligned}
\Delta_{11}= & \frac{1}{A_{1}} \frac{\partial u_{1}}{\partial \alpha_{1}}+\frac{u_{2}}{A_{1} A_{2}} \frac{\partial A_{1}}{\partial \alpha_{2}}-\frac{u_{3}}{R_{1}}, \\
\Delta_{22}= & \frac{u_{1}}{A_{1} A_{2}} \frac{\partial A_{2}}{\partial \alpha_{1}}+\frac{1}{A_{2}} \frac{\partial u_{2}}{\partial \alpha_{2}}-\frac{u_{3}}{R_{2}}, \\
\Delta_{12}= & \frac{1}{A_{2}} \frac{\partial u_{1}}{\partial \alpha_{2}}-\frac{u_{1}}{A_{1} A_{2}} \frac{\partial A_{1}}{\partial \alpha_{2}} \\
& +\frac{1}{A_{1}} \frac{\partial u_{2}}{\partial \alpha_{1}}-\frac{u_{2}}{A_{1} A_{2}} \frac{\partial A_{2}}{\partial \alpha_{1}} ;
\end{aligned}
$$

$\chi_{11}=-\frac{1}{A_{1} R_{1}} \frac{\partial u_{1}}{\partial \alpha_{1}}-\frac{u_{2}}{A_{1} A_{2} R_{2}} \frac{\partial A_{1}}{\partial \alpha_{2}}+\frac{1}{A_{1}^{3}} \frac{\partial A_{1}}{\partial \alpha_{1}} \frac{\partial u_{3}}{\partial \alpha_{1}}$

$-\frac{1}{A_{1}^{2}} \frac{\partial^{2} u_{3}}{\partial \alpha_{1}^{2}}-\frac{1}{A_{1} A_{2}^{2}} \frac{\partial A_{1}}{\partial \alpha_{2}} \frac{\partial u_{3}}{\partial \alpha_{2}}$,

$\chi_{22}=-\frac{u_{1}}{A_{1} A_{2} R_{1}} \frac{\partial A_{2}}{\partial \alpha_{1}}-\frac{1}{A_{2} R_{2}} \frac{\partial u_{2}}{\partial \alpha_{2}}$

$+\frac{1}{A_{2}^{3}} \frac{\partial A_{2}}{\partial \alpha_{2}} \frac{\partial u_{3}}{\partial \alpha_{2}}-\frac{1}{A_{2}^{2}} \frac{\partial^{2} u_{3}}{\partial \alpha_{2}^{2}}-\frac{1}{A_{1}^{2} A_{2}} \frac{\partial A_{2}}{\partial \alpha_{1}} \frac{\partial u_{3}}{\partial \alpha_{1}}$,

$\chi_{12}=-\frac{1}{A_{2} R_{1}} \frac{\partial u_{1}}{\partial \alpha_{2}}+\frac{u_{1}}{A_{1} A_{2} R_{1}} \frac{\partial A_{1}}{\partial \alpha_{2}}-\frac{1}{A_{1} R_{2}} \frac{\partial u_{2}}{\partial \alpha_{1}}$

$+\frac{u_{2}}{A_{1} A_{2} R_{2}} \frac{\partial A_{2}}{\partial \alpha_{1}}+\frac{1}{A_{1}^{2} A_{2}} \frac{\partial A_{1}}{\partial \alpha_{1}} \frac{\partial u_{3}}{\partial \alpha_{2}}$

$+\frac{1}{A_{1} A_{2}^{2}} \frac{\partial A_{2}}{\partial \alpha_{2}} \frac{\partial u_{3}}{\partial \alpha_{1}}-\frac{2}{A_{1} A_{2}} \frac{\partial^{2} u_{3}}{\partial \alpha_{1} \partial \alpha_{2}}$

$$
+\frac{1}{A_{1}^{2} A_{2}} \frac{\partial A_{1}}{\partial \alpha_{2}} \frac{\partial u_{3}}{\partial \alpha_{1}}+\frac{1}{A_{1} A_{2}^{2}} \frac{\partial A_{2}}{\partial \alpha_{1}} \frac{\partial u_{3}}{\partial \alpha_{2}} .
$$

These are the most general thin shell geometric equations.

The equations (4) and (5) can be converted to shells of specific geometric shapes by substituting in them the corresponding values of the geometrical functions.

\section{Geometric functions}

For clarity, we write out the most common way of defining the parametres $\alpha_{1}, \alpha_{2}$ and the resulting values of the functions (scale factors) $A_{1}, A_{2}$ for the types of shells of usual practical interest.

Fig. 3 shows the simplest case of a flat plate using rectangular coordinates $\alpha_{1}=x, \alpha_{2}=y$, for which the scale factors $A_{1}=1$ and $A_{2}=1$.

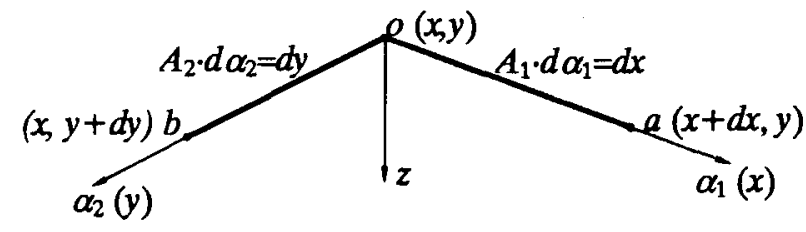

Fig. 3. Flat plate

In Fig. 4, using polar coordinates $\alpha_{1}=r, \alpha_{2}=\theta$, the same is true in the radial direction, $A_{1}=1$. In the angular direction, a small change $d \theta$ in the parameter $\alpha_{2}=\theta$ produces an arc length of $r d \theta=A_{2}$ $d \alpha_{2}$ giving $A_{2}=r$.

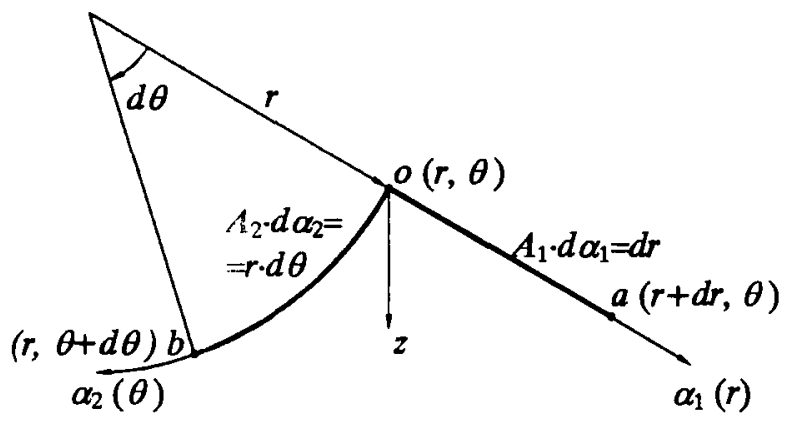

Fig. 4. Axi-rotational plate

Fig. 5 for the right cylindrical shell, using an angular coordinate, seems equally clear. In this case $\alpha_{1}=x, \alpha_{2}=\theta, A_{1}=1$ and $A_{2}=R_{2}$.

Fig. 6 shows the case of a conical shell, using distances along the axis $x=\alpha_{1}$, and rotations about the axis $\theta=\alpha_{2}$. In the $x$ direction the actual distances along the middle surface are $x / \cos \gamma$, where $\gamma$ is the cone angle; hence the scale factor $A_{1}=1 / \cos \gamma$. In the $\alpha_{2}=\theta$ direction the length of the $\operatorname{arc} a b$ is $A_{2} d \alpha_{2}=r d \theta$ $=x \operatorname{tg} \gamma d \theta$ and the scale factor $A_{2}=x \operatorname{tg} \gamma$. 


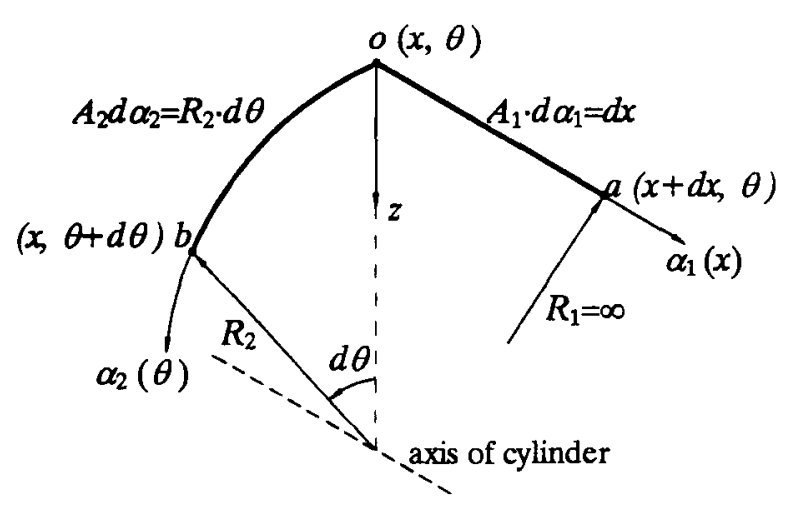

Fig. 5. Cylindrical shell

In the case of the axi-rotational shell, shown in Fig. 7, we take the coordinates as two angles, one angle of latitude $\alpha_{1}=\varphi$, and the second angle of longitude $\alpha_{2}=\theta$. Then the scale factors $A_{1}=R_{1}$ and $A_{2}=r=R_{2} \sin \varphi$ Here $R_{1}, R_{2}$ are the radii of curvatures in the $\alpha_{1}, \alpha_{2}$ directions.

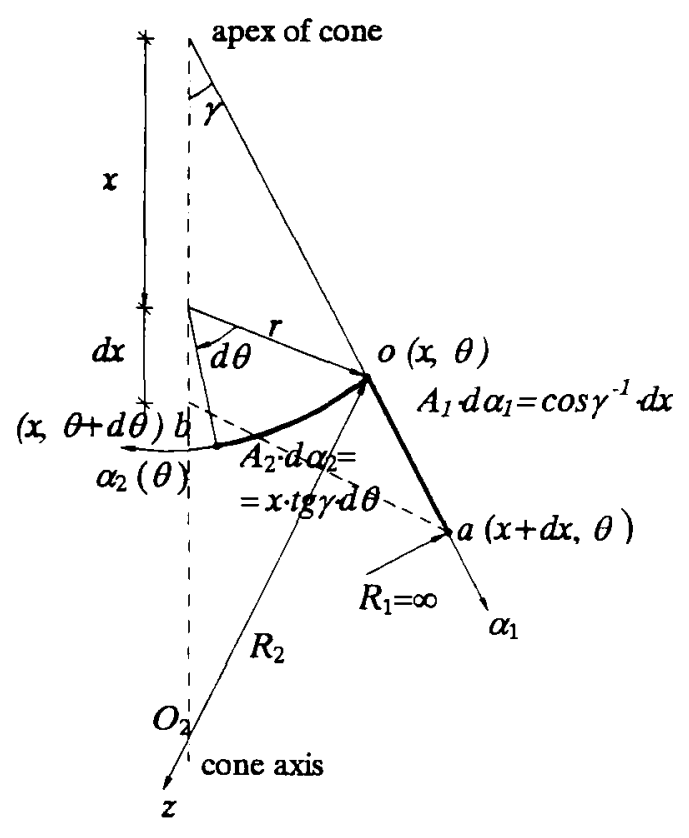

Fig. 6. Circular cone

Fig. 8 shows a double-curved shell, using rectangular coordinates, for which the scale factor $A_{1}=1$ and $A_{2}=1$.

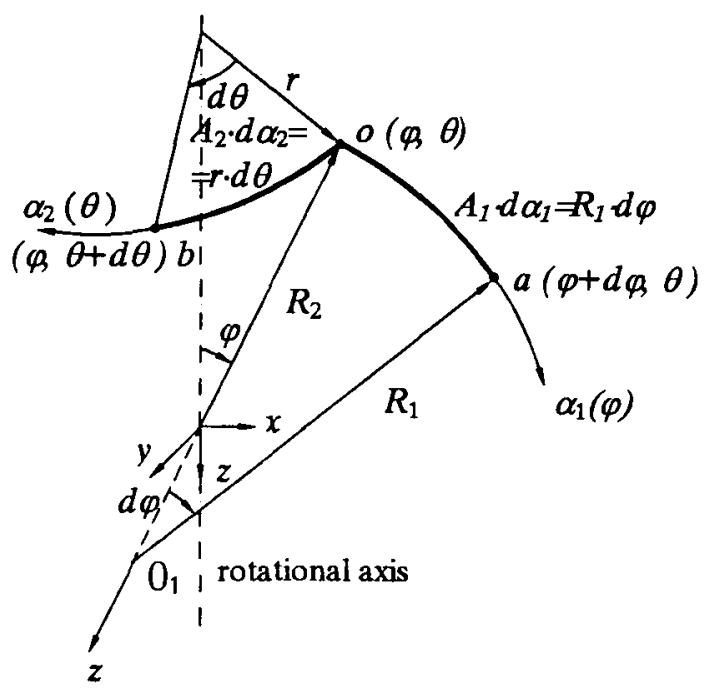

Fig. 7. Axi-rotational shell

In all these cases, the same resulting values of scale factors may be given, using the Eqs. (1). The equations of middle surface is then found as a continuous functions of $\alpha_{1}, \alpha_{2}$. For example, in the case of sphere (see Fig. 7 with $R_{1}=R_{2}=R$ ) equations of middle surface are:

$x=R \sin \varphi \cos \Theta, \quad y=R \sin \varphi \sin \Theta, \quad z=-R \cos \varphi$.

Then

$$
\begin{aligned}
& A_{1}=\sqrt{(R \cos \varphi \cos \Theta)^{2}+(R \cos \varphi \sin \Theta)^{2}+(R \sin \varphi)^{2}}=R, \\
& A_{2}=\sqrt{(R \sin \varphi \sin \Theta)^{2}+(R \sin \varphi \cos \Theta)^{2}}=R \sin \varphi .
\end{aligned}
$$

These values are defined in Fig. 7.

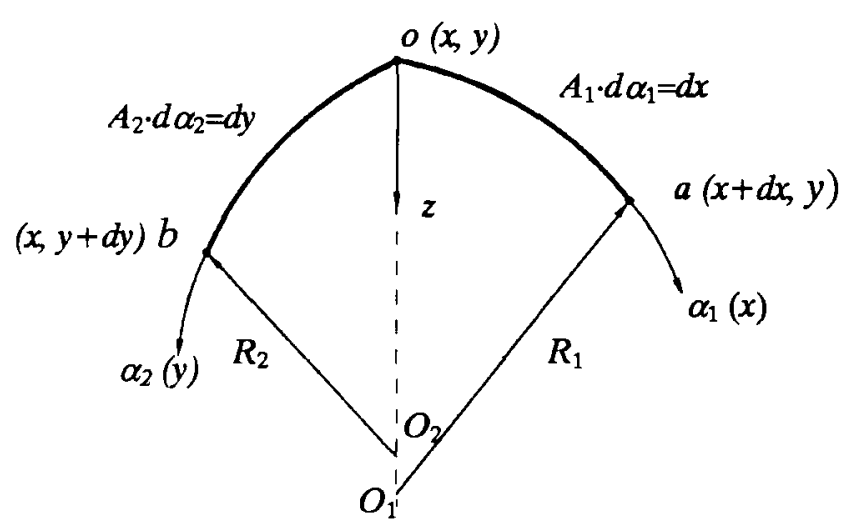

Fig. 8. Double-curved (general) shell 


\section{Conchusions}

Equations (4) and (5) together with Eqs. (2) form the dual relations for general thin shell linear theory. They, and modifications of them, can be converted to theories for shells of specific geometric shapes merely by substituting in them the corresponding values of the geometric functions, such as those discussed previously.

\section{References}

1. A.E.Love. A Treatise on the Mathematical Theory of Elasticity. $4^{\text {th }}$ ed.-Cambridge: University Press, 1927. $517 \mathrm{p}$.

2. А.P.Рхханищын. Двойственность стятнческих и геометрических уравнений стронтельной механики // Строитељьство и архитехтура, Известия высших учебных заведенпй, 스 11, 1974, с. 34-41.

Iteikta 19970120
DUALIOS PRIKLAUSOMYBÉ TIESINEJE PLONU APIBENDRINTOS FORMOS KEVALU TEORIJOJE

\section{R. Karkauskas}

\section{S a n tra u k a}

Straipsnyje naudojant Kirchofo-Liavo hipoteze išvestos plono apibendrintos formos kevalo elemento dualios priklausomybès. Jas sudaro diferencialinès pusiausvyros ir geometrinès lygtys kreivalinijinéje koordinaxiy sistemoje. Pateiktos priklausomybiu kvadratinès formos koeficienty reikšmès j̇vairiose koordinačiy sistemose, kurios gali būti taikomos konkrečios formos kevalams skaičiuoti.

Romangs KARKaUSKaS. Doctor, Associate Professor. Department of Structural Mechanics. Vilnius Gediminas Technical University (VGTU). 11 Saulètekio Ave, 2040 Vilnius, Lithuania. Dr degree in 1972 (structural mechanics). Scientific visits: Warsaw Politechnical Institute, Moscow Civil Engineering Institute, Kiev Civil Engineering Institute. Research interests: analysis and optimization of elastic-plastic structures, computational mechanies. 